\title{
Interaction of Psychopathic Traits Dimensions in the Prediction of Psychological and Physical Child-to-Parent Violence in Adolescents
}

\author{
Joana Del Hoyo-Bilbao ${ }^{1}$ (D) $\cdot$ Izaskun Orue $^{1}$ (D) $\cdot$ Esther Calvete $^{1}$ (D)
}

Accepted: 14 October 2021 / Published online: 4 November 2021

(c) The Author(s) 2021

\begin{abstract}
This study examined the longitudinal relationships between three psychopathic trait dimensions (callous-unemotional, CU; grandiose-manipulative, GM; and impulsive-irresponsible, II) and their interaction in the prediction of psychological and physical child-to-parent violence (CPV). Furthermore, the study examined whether the predictive relationships were different for boys and girls. A total of 765 adolescents ( 463 girls), with a mean age of 15.28 years $(S D=1.04)$, completed measures of the psychopathic trait dimensions (CU, GM, and II) and psychological and physical CPV at the beginning of the study and six months later. At the cross-sectional level, all the psychopathic trait dimensions were related to psychological and physical CPV, although the effect sizes were small. At the longitudinal level, II predicted increased psychological CPV. A significant three-way interaction between the three psychopathic trait dimensions (CU, GM, and II) indicated that the longitudinal association between II and physical CPV was higher for adolescents with high CU and low GM. However, the predictive model did not differ for boys and girls.
\end{abstract}

Keywords Child-to-parent violence · Aggression · Psychopathic trait dimensions · Callous-unemotional · Grandiosemanipulative $\cdot$ Impulsive-irresponsible

\section{Introduction}

Child-to-parent violence (CPV) is a type of family violence characterized by repeated physical, psychological (verbal or non-verbal), or economic violence directed toward one's parents or those who fill parenting roles (Pereira et al., 2017). According to epidemiological research, the prevalence of CPV is relatively high, especially for psychological violence. One study reported rates of $14.2 \%$ and $3.2 \%$ for repeated psychological and physical CPV last year, respectively, in a community sample of 2672 Spanish adolescents (Calvete et al., 2013). Moreover, the prevalence of CPV is generally higher among offender and clinical samples (Ibabe \& Jaureguizar, 2010; Purcell et al., 2014).

Joana Del Hoyo-Bilbao

joana.delhoyo@deusto.es

Izaskun Orue

izaskun.orue@deusto.es

Esther Calvete

esther.calvete@deusto.es

1 Department of Psychology, Avenida de Las Universidades

24, University of Deusto, 48080 Bilbao, Spain
To develop adequate preventive interventions, it is necessary to identify the factors that lead to CPV. Previous studies have suggested that multiple factors are involved in the development and continued perpetration of CPV, including substance use (Calvete et al., 2012), family characteristics (Ibabe \& Bentler, 2016), and exposure to violence (Boxer et al., 2009; Calvete et al., 2015b). In a recent review study, the authors concluded that CPV often appears to be part of a pattern of antisocial or aggressive behavior, in which adolescents who are aggressive toward their parents tend to display aggressive behavior in general (Simmons et al., 2018). However, they also highlighted that there is limited information on the role of individual characteristics, such as cognitive and emotional factors, relevant in general aggressive behavior regarding CPV.

Psychopathic traits have been proposed as important dispositional characteristics associated with aggressive behavior. Recently, the study of psychopathic traits has been extended to the child and adolescent population (for a review, see Salekin, 2017) to explore how these personality traits develop and analyze the relationships they can have with aggressive behavior. Findings from different studies indicated that psychopathic structure in childhood and adolescence is very similar to that in adults. Further, more 
recent studies have suggested that psychopathic structure is organized in a continuum rather than in categories representing the presence or absence of psychopathy (Marcus et al., 2004; Salekin, 2017).

Some studies have used factor analysis to examine the dimensionality of psychopathy in children and adolescents (Andershed et al., 2002; Frick et al., 2000; Van Baardewijk et al., 2010) and proposed that psychopathic personality traits among children and adolescents comprise three interrelated dimensions: affective, interpersonal, and behavioral. The interpersonal dimension is characterized by grandiosity, superficial charm, and egocentricity (grandiose-manipulative trait dimension; GM); the affective dimension is represented by remorselessness, callousness, and lack of empathy (callous-unemotional trait dimension; $\mathrm{CU}$ ); and the behavioral dimension implies impulsiveness and irresponsibility (impulsiveness-irresponsibility trait dimension; II). Studies have consistently found a relationship between psychopathic trait dimensions and externalizing behaviors, such as antisocial and delinquent behavior (Penney \& Moretti, 2007; Somma et al., 2018), overt and relational aggression (Orue et al., 2016), bullying and cyberbullying (López-Larrañaga \& Orue, 2019; Orue \& Calvete, 2019), drug use (Hillege et al., 2010), or intimate partner violence (Kiire, 2017).

Until recently, studies have pointed to $\mathrm{CU}$ as the most powerful psychopathic dimension for explaining conduct problems at all ages, including children and adolescents (Frick \& White, 2008; Frick et al., 2005). However, several studies have recently highlighted that studying only $\mathrm{CU}$ to explain conduct problems is insufficient (Colins et al., 2018; Salekin 2016a, b, 2017). Thus, some studies have stressed the importance of studying the interaction between the three psychopathic dimensions to predict externalizing problems to better understand adolescents with conduct problems (Lilienfeld, 2018; Salekin, 2017). For example, Colins et al. (2018) compared two models of psychopathy to predict future and stable conduct problems. The first model included only the CU trait dimension, whereas the second was a multidimensional model that included three psychopathic trait dimensions (CU, II, and GM). The results indicated that the subtyping approach using various psychopathic traits dimensions was more informative in predicting future and stable conduct problems among children and adolescents. Moreover, in another study, Somma et al. (2018) found that the interaction of the three-trait dimensions improved the prediction of delinquent behavior in a sample of adolescents beyond the information provided by a single underlying psychopathic dimension. Similarly, Colins et al. (2014) found that the interaction of the psychopathic trait dimensions was more useful in predicting the occurrence of behavioral problems than evaluating only one of them. Specifically, they found that combining high scores in the three dimensions better predicted the occurrence of behavioral problems. Further, Fanti et al. (2018) found that combining high initial levels of conduct disorder symptoms and the CU trait dimension-as well as combining conduct disorder symptoms, GM, and II—can predict later clinically significant conduct disorder symptoms. Finally, the interaction between the psychopathic trait dimensions has also been shown to be significant in predicting bullying and cyberbullying behavior (López-Larrañaga \& Orue, 2019; Orue \& Andershed, 2015).

Although the association between the psychopathic trait dimensions and CPV has not specifically been examined, some previous findings have suggested that these trait dimensions could be related to this type of aggressive behavior. In addition, some cross-sectional studies have found a positive relationship between impulsivity and CPV (Calvete et al., 2011; Del Hoyo-Bilbao et al., 2020; Rico et al., 2017). In analyzing the personal files of young offenders who committed CPV, Ibabe and Jaureguizar (2010) found a lack of empathy in these adolescents. Calvete et al. (2015b) found that narcissism predicted CPV. Additionally, other types of aggressive behaviors are commonly present among adolescents who enact CPV (Pagani et al., 2004, 2009; Simmons et al., 2018). As a result, psychopathic trait dimensions could have a role in $\mathrm{CPV}$.

\section{Sex Differences in CPV and Psychopathic Trait Dimensions}

The differences between girls and boys in the perpetration of CPV are not clear. Some studies conducted in community and forensic samples did not find significant differences between boys and girls (Del Hoyo-Bilbao et al., 2018b; Gámez-Guadix \& Calvete, 2012; Pagani et al., 2004, 2009). However, other studies' results suggest that, in community samples, girls tend to commit more psychological aggression than boys (Calvete \& Orue, 2016; Lyons et al., 2015; Margolin \& Baucom, 2014). Furthermore, some studies have indicated that, in judicial contexts, boys perpetrate higher levels of physical CPV (Boxer et al., 2009; Ghanizadeh \& Jafari, 2010; Routt \& Anderson, 2011), whereas, in community samples, girls perpetrate more physical CPV than boys (Calvete et al., 2013, 2015b). However, other studies found no differences in physical aggression between boys and girls in judicial samples and found that girls show higher levels of relational aggression (Stickle et al., 2012) and physical CPV (Strom et al., 2014). There is a greater consensus on sex differences in psychopathic traits. Several studies have indicated higher scores among adolescent boys in GM and CU trait dimensions (Andershed et al., 2018; Fanti \& Kimonis, 2012; Fanti et al., 2009). However, in the II trait dimension, some studies have found higher scores in boys (Andershed et al., 2018; Colins et al., 2018; Fanti et al., 2009), whereas others have reported higher scores in girls (Orue et al., 2016). There are no studies on sex differences in 
the associations between psychopathic trait dimensions and CPV. Nevertheless, some studies have examined sex differences in the relationship between psychopathic trait dimensions and other types of aggressive behavior. Furthermore, some studies have indicated the absence of sex differences in the associations between psychopathic traits and aggression and delinquency (Marsee et al., 2005; Penney \& Moretti, 2007). However, other studies found sex differences when considering different modalities of aggressive behavior. For example, in a study with adolescents, the $\mathrm{CU}$ trait dimension predicted proactive aggression only in girls, whereas the GM trait dimension predicted proactive aggression only in boys (Orue et al., 2016).

\section{The Present Study}

It is important to identify individual variables related to CPV development (Calvete et al., 2012; Simmons et al., 2018). The similarity between psychopathic trait dimensions and some individual characteristics of adolescents who commit CPV indicates a possible relationship between II, GM, and $\mathrm{CU}$ trait dimensions and CPV. Thus, this study first aim to analyze the longitudinal relationships between psychopathic trait dimensions (II, GM, CU) and psychological and physical CPV perpetration. Furthermore, according to previous studies, the interaction of psychopathic trait dimensions can provide more useful information than the isolated role of each trait dimension (Colins et al., 2014; Lilienfeld, 2018). Therefore, the second purpose of this study is to examine the interaction of the psychopathic trait dimensions in predicting psychological and physical CPV. Finally, given the sex differences found in previous studies on CPV, the psychopathic trait dimensions, and the relationships between the psychopathic trait dimensions and aggression, the third objective is to examine whether the predictive model is similar in boys and girls.

\section{Method}

\section{Participants}

The initial sample comprised 1002 high school students from 13 randomly selected schools in Bizkaia (Spain). The final sample comprised 765 adolescents (463 girls and 302 boys) who completed measures at both Time 1 (T1) and Time 2 (T2). All the participants completed the instrument at $\mathrm{T} 1$ and $\mathrm{T} 2$ six months apart. There were differences in some of the study variable means at $\mathrm{T} 1$ between the adolescents who completed both waves and those who failed to complete the study. Specifically, the means of adolescents who failed to complete the study were higher in CU 3.29 $(S D=3.60)$ vs. $2.77(S D=2.92)$; $t(341.38)=2.05, p=0.041$;
II $6.63(S D=4.02)$ vs. $5.97(S D=3.40) ; t(350.76)=2.30$, $p=0.022)$ and physical CPV $0.08(S D=0.29)$ vs. 0.04 $(S D=0.16) ; t(280.72)=2.04, p=0.043$. Participants were between 14 and 19 years old $\left(M_{\text {age }}=15.28\right.$ years, $\left.S D=1.04\right)$, and $91.2 \%$ were Spanish, $6.2 \%$ were Latin American, $0.7 \%$ were African, $0.6 \%$ were Eastern European, and $1.3 \%$ came from other regions. To evaluate participants' socioeconomic levels, the criteria recommended by the Spanish Society of Epidemiology (2000) were used, and the distribution was as follows: $17.1 \%$ low, $27.6 \%$ medium-low, $27.1 \%$ medium, $14.6 \%$ medium-high, and $13.7 \%$ high.

\section{Measures}

\section{Child-to-Parent Violence}

CPV was measured using the Child-to-Parent Aggression Questionnaire (CPAQ; Calvete et al., 2013). The CPAQ comprises 20 items, 10 referring to fathers and 10 referring to mothers. Of the 10 items relevant to each parent, three assess physical aggression and seven assess psychological aggression. Participants reported how often they performed each aggressive act against their parents using a 4-point scale ranging from 0 (never) to 3 (six or more times). The CPAQ has previously shown excellent psychometric properties (Calvete \& Orue, 2016). In this study, Cronbach's alpha coefficients were 0.62 and 0.73 for physical CPV and 0.86 and 0.88 for psychological CPV at T1 and T2, respectively.

\section{Psychopathic Traits Dimensions}

The Youth Psychopathic Inventory-Short Form (YPI-S; Van Baardewijk et al., 2010) was used to measure psychopathic trait dimensions. The scale evaluates three psychopathic dimensions with six items per dimension: the GM, CU, and II trait dimensions. The items were rated on a 4-point Likert-type scale ranging from 1 (does not apply at all) to 4 (applies very well). The Spanish version was used in this study, and it showed good psychometric properties as well (Orue \& Andershed, 2015). Cronbach's alphas were 0.82, 0.69 , and 0.71 for GM, CU, and II, respectively.

\section{Procedure}

The schools used in this study were chosen at random. First, permission was obtained from the schools. Second, all parents were informed of the nature of the study and its voluntary, anonymous, and confidential nature through an informed consent form. They were informed that their children's participation in the study would not cause them any harm. Third, the researchers' contact information was provided for any clarification or questions they might have. Finally, they were invited to refuse their children's 
participation through the informed consent form. Only two parents refused to allow their children to be included in the study. Participants received the same information as their parents, and all adolescents consented to participate in the study. Data were collected on two occasions with a 6-month interval. To preserve each participants' identity, codes were used to pair the questionnaires from T1 and T2. All data were recorded under the code of ethics in research and kept confidential. Preacher and Coffman's (2006) calculator was used to perform a power analysis. The power in the study was $99.58 \%$ for a sample of 765 participants. The study and its consent procedure were reviewed and approved by the Ethics Committee of the University [masked].

\section{Data Analysis}

To assess the longitudinal relationships between the psychopathic dimensions and CPV, a paths analysis was conducted via the robust maximum likelihood (ML) estimation method with the Satorra-Bentler scale chi-square (S-B $\chi 2$ ) with LISREL 8.8 (Jöreskog \& Sörbom, 2006). The hypothesized model included GM, CU, and II measures at T1 and psychological and physical CPV at T1 and T2. The model included autoregressive paths between psychological and physical CPV at $\mathrm{T} 1$ and $\mathrm{T} 2$. The model also included paths from the interaction terms among the psychopathic dimensions $(\mathrm{GM} \times \mathrm{CU}$, $\mathrm{GM} \times \mathrm{II}$, and $\mathrm{CU} \times \mathrm{II})$ and the three-way interaction term among the three psychopathic dimensions $(\mathrm{GM} \times \mathrm{CU} \times \mathrm{II})$. The study followed standard procedures to create the moderation terms and transformed all the psychopathic trait dimensions into z-scores. The procedures proposed by Dawson (2018) were followed to examine the differences between previously plotted slopes once the interaction terms were significant. To examine whether the data adjusted adequately to the model, the following criteria were used to assess goodnessof-fit: CFI values of 0.95 or greater, NNFI values of 0.90 or greater, and RMSEA values of 0.08 or less indicated that the model fits the data adequately (Byrne, 2006).

\section{Results}

\section{Descriptive Analyses and Sex Differences}

Table 1 presents descriptive statistics (mean and $S D$ ) and Pearson correlations for the study variables. All correlations were significant except the correlation between $\mathrm{CU}$ and psychological CPV at T2 $(p=0.08)$. However, $\mathrm{CU}$ and psychological CPV at $\mathrm{T} 1$ were positively statistically associated, but the correlation was small. All other psychopathic dimensions were positively associated with psychological and physical CPV at T1 and T2. All correlations were small, except for the correlations between II and psychological $\mathrm{CPV}$ at $\mathrm{T} 1$ and $\mathrm{T} 2$, which were moderate. The psychopathic trait dimensions were also positively related, indicating that high levels in one dimension were related to high levels in the other dimensions. The correlations between trait dimensions were moderate, except for the correlation between II and CU, which was small. Psychological and physical CPV at T1 and T2 were positively and moderately related, except for psychological CPV at T1 and T2, which was highly correlated. This result indicates the stability of the CPV measurement. Table 2 presents sex differences for all the study variables. Girls obtained higher scores on the II trait dimension $(d=0.24)$ and psychological $(d=0.61)$ and physical CPV $(d=0.20)$ at T1 and on psychological CPV at T2 $(d=0.54)$. Boys obtained higher scores on $\mathrm{CU}(d=0.38)$ and $\mathrm{GM}$ trait dimensions $(d=0.23)$. There were no differences between girls and boys in physical CPV at T2 $(d=0.08)$. Therefore, the effect sizes evaluated with Cohen's $d$ metric were small for all differences, except for differences in psychological CPV at both $\mathrm{T} 1$ and $\mathrm{T} 2$, which were found to be medium.

\section{Predictive Model}

First, the hypothesized path analysis model was estimated, including all the relationships between the study variables,
Table 1 Descriptive statistics and correlations among the study variables

\begin{tabular}{llllllll}
\hline & 1 & 2 & 3 & 4 & 5 & 6 & 7 \\
\hline 1. Callous-Unemotional & 1 & & & & & & \\
2. Grandiose-Manipulative & $.38^{* * *}$ & 1 & & & & & \\
3. Impulsive-Irresponsible & $.28^{* * *}$ & $.37 * * *$ & 1 & & & & \\
4. Psychological CPV T1 & $.10^{* * *}$ & $.21^{* * *}$ & $.40^{* * *}$ & 1 & & & \\
5. Physical CPV T1 & $.08^{*}$ & $.09^{* *}$ & $.18^{* * *}$ & $.40^{* * *}$ & 1 & & \\
6. Psychological CPV T2 & .06 & $.19^{* * *}$ & $.40^{* * *}$ & $.74 * * *$ & $.35^{* * *}$ & 1 & \\
7. Physical CPV T2 & $.07 *$ & $.08^{*}$ & $.15^{* * *}$ & $.34 * * *$ & $.47 * * *$ & $.36^{* * *}$ & 1 \\
$M$ & 2.77 & 3.26 & 5.98 & 0.56 & 0.38 & 0.56 & 0.03 \\
$S D$ & 2.95 & 3.60 & 3.41 & 0.49 & 0.16 & 0.47 & 0.13 \\
\hline$*$ & & & & & & &
\end{tabular}


Table 2 Sex Differences for all variables of the study

\begin{tabular}{|c|c|c|c|c|c|c|c|}
\hline & \multicolumn{2}{|c|}{ Girls $(N=463)$} & \multicolumn{2}{|c|}{ Boys $(N=302)$} & \multirow[b]{2}{*}{$t$} & \multirow[b]{2}{*}{$p$} & \multirow[b]{2}{*}{ Cohen's $d$} \\
\hline & Mean & $S D$ & Mean & $S D$ & & & \\
\hline Callous-Unemotional & 2.33 & 2.73 & 3.45 & 3.14 & -5.08 & .000 & 0.38 \\
\hline Grandiose-Manipulative & 2.92 & 3.22 & 3.78 & 4.07 & -3.12 & .002 & 0.23 \\
\hline Impulsive-Irresponsible & 6.30 & 3.37 & 5.47 & 3.43 & 3.28 & .001 & 0.24 \\
\hline Psychological CPV T1 & 0.67 & 0.52 & 0.39 & 0.38 & 8.45 & .000 & 0.61 \\
\hline Physical CPV T1 & 0.05 & 0.19 & 0.02 & 0.09 & 3.24 & .001 & 0.20 \\
\hline Psychological CPV T2 & 0.66 & 0.49 & 0.42 & 0.40 & 7.46 & .000 & 0.54 \\
\hline Physical CPV T2 & 0.03 & 0.15 & 0.02 & 0.09 & 1.32 & .187 & 0.08 \\
\hline
\end{tabular}

and the fit indices were: $\mathrm{S}-\mathrm{B} \chi^{2}(12, N=765)=102.06$, RMSEA $=0.099,90 \%$ CI $[0.082,0.12], \mathrm{CFI}=0.97$, $\mathrm{NNFI}=0.87$. Second, the nonsignificant paths were eliminated to create a more parsimonious model. The parsimonious predictive model (Fig. 1) obtained adequate fit indices: $\mathrm{S}-\mathrm{B} \chi^{2}(31, N=765)=161.85$, RMSEA $=0.074$, $90 \%$ CI $[0.063,0.086], \mathrm{CFI}=0.96, \mathrm{NNFI}=0.93$. The model explained $56 \%$ of the variance for psychological $\mathrm{CPV}$ at $\mathrm{T} 2$ and $26 \%$ of the variance for physical CPV at $\mathrm{T} 2$.
Fig. 1 Psychopathic dimensions and interactions that predict Psychological and Physical CPV

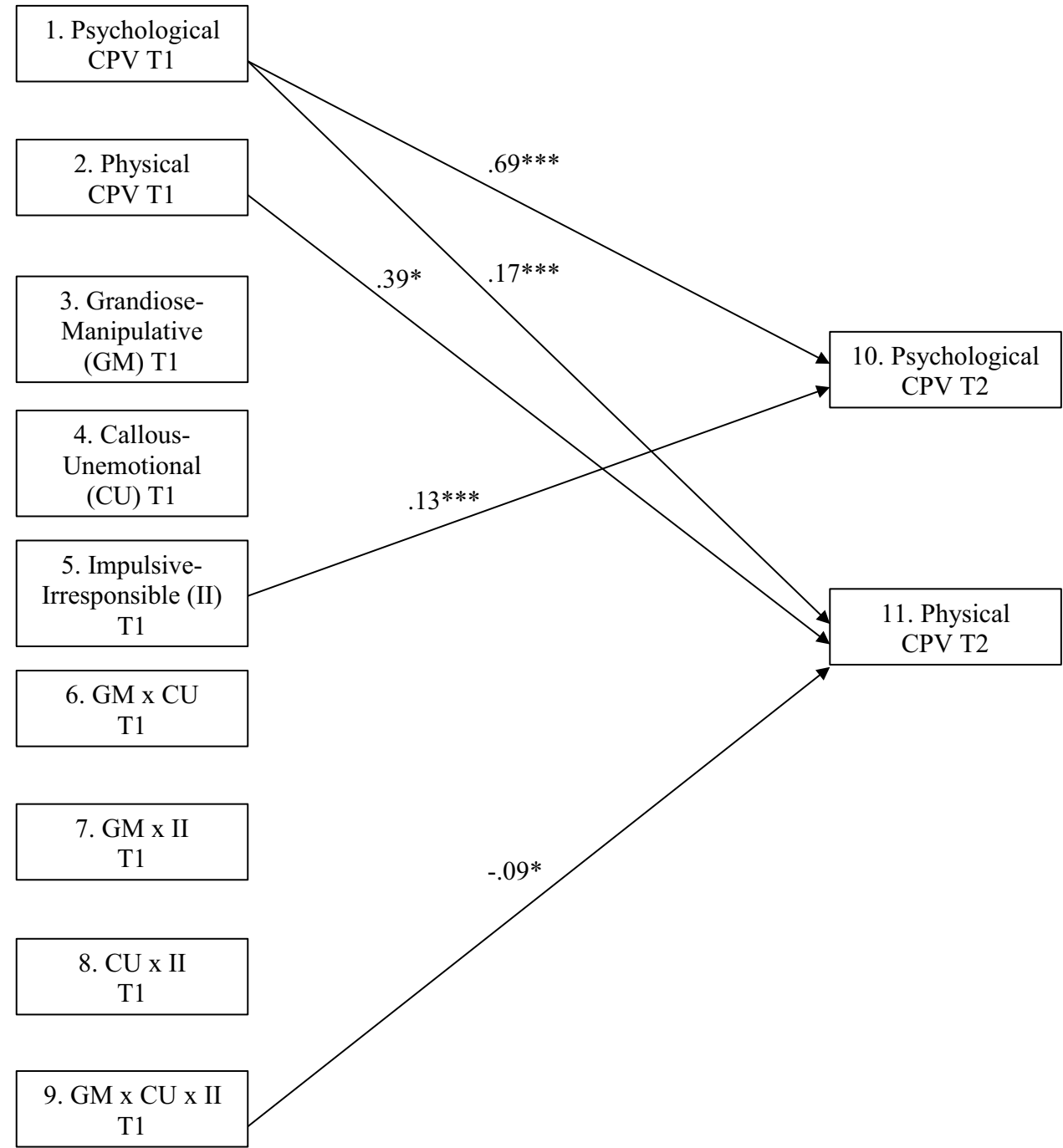

Note. Only statistically significant paths are shown. ${ }^{*} p<.05 ; * * p<.01 ; * * * p<001$ 
All autoregressive effects were statistically significant, indicating the stability of CPV over time. Psychological CPV at $\mathrm{T} 1$ predicted physical CPV at T2. II at T1 predicted psychological CPV at T2. The other two psychopathic trait dimensions did not predict changes in CPV over time. Neither of the two-way interaction terms between the psychopathic trait dimensions was statistically significant for physical CPV at $\mathrm{T} 2(\mathrm{GM} \times \mathrm{CU}$ [0.00 (0.01); $p=0.984], \mathrm{GM} \times \mathrm{II}$ [0.00 (0.01); $p=0.603]$, and $\mathrm{CU} \times \mathrm{II}[0.01(0.01) ; p=0.211])$. However, the interaction of the three psychopathic trait dimensions at $\mathrm{T} 1(\mathrm{GM} \times \mathrm{CU} \times \mathrm{II})$ predicted physical $\mathrm{CPV}$ at $\mathrm{T} 2$. Figure 2 represents the three-way interaction. The results indicated that II predicts CPV most when it is combined with high $\mathrm{CU}$ and low GM. The examination of the slopes indicated that II was only related to physical CPV when CU was high (Slope 1 [High GM], $\beta=0.017, t=2.95, p=0.003$ and Slope 2 [Low GM], $\beta=0.027, t=2.82, p=0.005$ ). II was not related to physical CPV when CU was low (Slope 3 [High $\mathrm{GM}$ ], $\beta=0.007, t=0.76, p=0.44$ and Slope 4 [Low GM], $\beta=-0.011, t=-1.41, p=0.16)$. Furthermore, the results indicated that the slope difference between (1) and (2) was significant, $t=-1.97, p=0.049$, indicating that, when $\mathrm{CU}$ is high, the impact of II on physical CPV is higher when GM is low than when it is high. Further, the slope differences between (1) and (4) and between (2) and (4) were statistically significant $(t=2.27, p=0.024$ and $t=3.73 ; p<0.001$, respectively), indicating that when $\mathrm{CU}$ is high (Slopes 1 and 2); the slope is higher than when both CU and GM are low (Slope 4).

\section{Sex Differences for the Predictive Model}

To examine whether the predictive model was sex invariant, we performed a multiple group analysis. For this purpose, the following steps were carried out. First, we estimated the model for girls and boys separately. The fit indices were adequate for girls, $\chi^{2}(30, N=463)=94.86$, RMSEA $=0.068$, $90 \%$ CI $[0.053,0.084], \mathrm{CFI}=0.95, \mathrm{NNFI}=0.92$ and for boys, $\chi^{2}(30, N=302)=100.37$, RMSEA $=0.088,90 \% \mathrm{CI}$ $[0.070,0.011], \mathrm{CFI}=0.96, \mathrm{NNFI}=0.92$. Second, the configural invariance of the model was tested to demonstrate that the pattern of the fixed and free parameters was equivalent across the subsamples, $\chi^{2}(60, N=765)=194.940$, RMSEA $=0.0768,90 \%$ CI [0.0649, 0.0889], CFI $=0.956$, $\mathrm{NNFI}=0.919, \mathrm{SRMR}=0.186$. Finally, we estimated a model in which longitudinal paths were constricted to be equal across both subsamples. This constriction did not increase $\chi^{2}$ significantly, $\Delta \chi^{2}(12, N=765)=11.145, p=0.516$, which indicates that the overall pattern of paths was similar for girls and boys.

\section{Discussion}

This study examined the longitudinal relationships between psychopathic trait dimensions (GM, CU, and II) and psychological and physical CPV in adolescents. Overall, the results showed that psychopathic trait dimensions had a minimal role in CPV. At the cross-sectional level, all three psychopathic trait dimensions were positively related to psychological and physical CPV, although the effect sizes were small. At the longitudinal level, only II predicted psychological CPV six months later. Moreover, the results indicated a second-order interaction between psychopathic trait dimensions, which reveals the importance of the conjoint action of these trait dimensions.

Regarding the role of II as a predictor of psychological CPV, Rico et al. (2017) also found a positive relationship between attentional impulsivity and psychological CPV, but
Fig. 2 Three-way interaction of Callous-unemotional, Grandiose-manipulative, and Impulsivity-irresponsibility traits in predicting Physical CPV

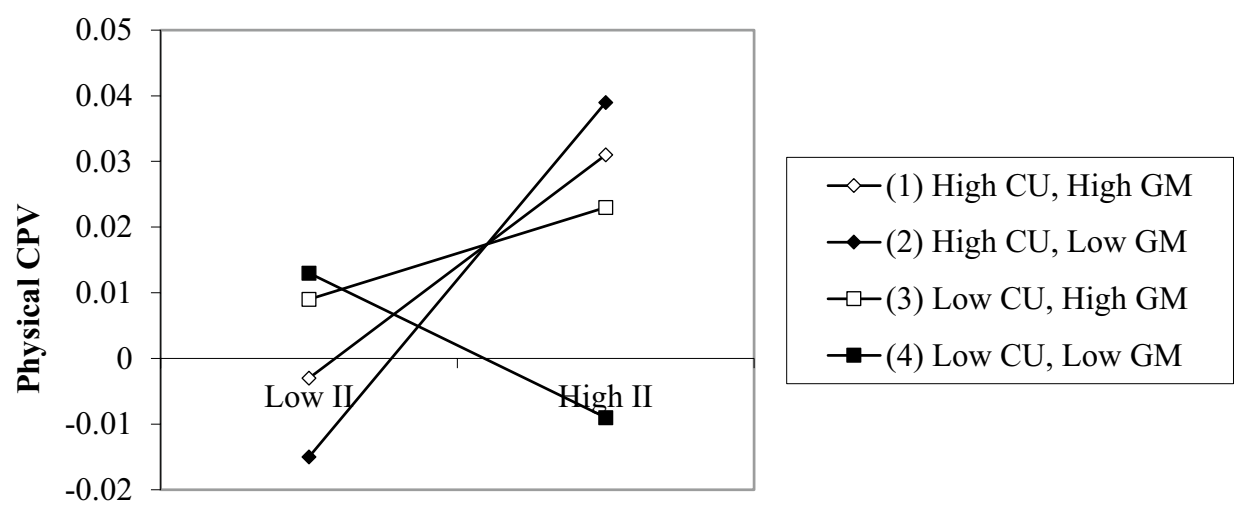

Note. CPV (child-to-parent violence); GM (grandiose-manipulative); CU (callous- 
not physical CPV. To interpret these results, an additional explanation is necessary. As a result, Rico et al. (2017) evaluated three dimensions of impulsivity: attentional impulsivity with a predominant component of cognitive or emotional instability; motor impulsivity in which motor activation prevails; and lack of planning with lack of self-control (Barratt et al., 1997). Thus, attentional impulsivity was related to psychological CPV, motor impulsivity was related to psychological and physical CPV, and lack of planning was not related to either type of CPV. The II evaluated in this study as a dimension of psychopathy has an important component of emotional instability (Andershed et al., 2002). Therefore, it seems that impulsiveness, which is understood as emotional instability or a lack of reflecting before acting, is imperative in predicting psychological aggression, such as insulting or making threats against one's parents, while for physical CPV, additional motor activation becomes more important.

Although simple trait dimension interactions were not related to either type of CPV, the three-way interaction of psychopathic trait dimensions predicted physical CPV. Thus, II is more strongly associated with physical CPV when concurrent with high levels of UC and low levels of GM. Furthermore, within this three-way interaction, the moderating role of CU is especially relevant, as when CU is low, II is not significantly associated with physical CPV. Overall, these results expand current knowledge on CU's role in the development of physical CPV. Thus, it seems that for physical $\mathrm{CPV}$, alongside the important roles of emotional instability or lack of reflection, lack of empathy or affective indifference in adolescents also plays a substantial role. This follows the findings of Ibabe and Jaureguizar (2010), who indicated that young offenders who committed CPV also lacked empathy. A possible explanation for this may be that adolescents in community samples who perpetrate physical CPV share more characteristics with adolescents in offender samples, in whom physical CPV levels tend to be much higher (Kennedy et al., 2010; Purcell et al., 2014).

The present results also indicated stability in CPV behaviors over time, consistent with previous studies (Calvete et al., 2015a). Furthermore, the perpetration of psychological CPV predicted physical CPV, supporting the idea that CPV has an escalation component (Pereira \& Bertino, 2009), with a tendency to worsen over time (Del Hoyo-Bilbao et al., 2018a). These data highlight the relevance of implementing preventive interventions.

Finally, regarding the sex differences in psychopathic trait dimensions, boys scored higher in the CU and GM trait dimensions. These results are consistent with previous studies (Andershed et al., 2018; Fanti et al., 2009). In the case of II, girls obtained higher scores, as has been highlighted in some studies (Orue et al., 2016). However, it must be noted that all the differences were small. Regarding CPV, girls scored higher in psychological CPV, and the effect size was moderate. These results are consistent with studies where girls exercise a greater degree of psychological CPV, especially in community samples (Calvete \& Orue, 2016; Lyons et al., 2015; Margolin \& Baucom, 2014). In this study, girls also perpetrated more physical CPV. This result is consistent with other studies related to community samples (Calvete et al., 2013, 2015b), although it contrasts studies conducted on judicial samples where there were either no differences (Stickle et al., 2012) or the boys presented more physical aggression (Boxer et al., 2009; Routt \& Anderson, 2011). However, the general pattern of associations was similar for boys and girls. These results are consistent with previous studies on the relationships between psychopathic trait dimensions and general aggression (Marsee et al., 2005; Penney \& Moretti, 2007).

\section{Limitations and Future Research}

In interpreting these results, certain limitations must be considered. First, although self-reports are among the most extensively used measures in studies on CPV and psychopathic traits (Calvete et al., 2012; López-Larrañaga \& Orue, 2019), parental measures could also provide a more rounded view of CPV and adolescents' individual traits (Calvete et al., 2017). Second, the participants belonged to a community sample in which physical CPV levels were very low. This could have directly influenced the relationships studied because it makes it difficult for the possible predictors to explain the outcome variance (Rico et al., 2017). Therefore, we need to study psychopathic trait dimensions in clinical or offender samples of adolescents in which the prevalence of physical CPV is higher (Del Hoyo-Bilbao et al., 2018b). Third, although this study provides valuable information on the individual characteristics of adolescents in the development of CPV, it would be interesting to study the possible moderating role of these variables in the relationships between contextual variables and CPV (e.g., exposure to family violence and CPV). In this way, more information could be provided on the role of personality traits in predicting CPV.

\section{Conclusions}

The results of this study indicate that psychopathic trait dimensions in community samples of adolescents play a limited role in CPV development. However, adolescents from offender samples commonly present a wider pattern of antisocial behavior (Kennedy et al., 2010). Thus, as psychopathic trait dimensions have a more relevant role in adolescents who display conduct disorder symptoms (Fanti et al., 2018), future studies should examine whether psychopathic 
trait dimensions are more relevant for CPV in clinical and offender samples. Finally, this study supports the importance of analyzing the interaction of the three psychopathic trait dimensions to create a better understanding of aggressive behaviors (Lilienfeld, 2018; Somma et al., 2018), including CPV.

Supplementary Information The online version contains supplementary material available at https://doi.org/10.1007/s10862-021-09940-w.

Acknowledgements This study could not have been conducted without the valuable collaboration of the adolescents and the funding of Eusko Jaurlaritza (IT982-16)

Funding Open Access funding provided thanks to the CRUE-CSIC agreement with Springer Nature.

Availability of Data and Material The data are available. https://osf.io/ pg27a/?view_only=68dae066372c4faeb98e293ef06d0713

\section{Declarations}

Consent to Participate Informed consent was obtained from legal guardians of the participants. In addition, verbal informed consent was obtained prior to the participation from all adolescents.

Conflict of Interest Joana Del Hoyo-Bilbao, Izaskun Orue and Esther Calvete declare that they have no conflict of interest.

Open Access This article is licensed under a Creative Commons Attribution 4.0 International License, which permits use, sharing, adaptation, distribution and reproduction in any medium or format, as long as you give appropriate credit to the original author(s) and the source, provide a link to the Creative Commons licence, and indicate if changes were made. The images or other third party material in this article are included in the article's Creative Commons licence, unless indicated otherwise in a credit line to the material. If material is not included in the article's Creative Commons licence and your intended use is not permitted by statutory regulation or exceeds the permitted use, you will need to obtain permission directly from the copyright holder. To view a copy of this licence, visit http://creativecommons.org/licenses/by/4.0/.

\section{References}

Andershed, H., Colins, O. F., Salekin, R. T., Lordos, A., Kyranides, M. N., \& Fanti, K. A. (2018). Callous-unemotional traits only versus the multidimensional psychopathy construct as predictors of various antisocial outcomes during early adolescence. Journal of Psychopathology and Behavioral Assessment, 40(1), 16-25. https://doi.org/10.1007/s10862-018-9659-5

Andershed, H., Kerr, M., Stattin, H., \& Levander, S. (2002). Psychopathic traits in non-referred youths: Initial test of a new assessment tool. In E. Blaauw \& L. Sheridan (Eds.), Psychopaths: Current international perspectives (pp. 131-158). Elsevier.

Barratt, E. S., Stanford, M. S., Kent, T. A., \& Felthous, A. (1997). Neuropsychological and cognitive psychophysiological substrates of impulsive aggression. Biological Psychiatry, 41, 1045-1061. https://doi.org/10.1016/S0006-3223(96)00175-8

Boxer, P., Gullan, R. L., \& Mahoney, A. (2009). Adolescents' physical aggression toward parents in a clinic-referred sample. Journal of
Clinical Child \& Adolescent Psychology, 38(1), 106-116. https:// doi.org/10.1080/15374410802575396

Byrne, B. M. (2006). Structural equation modeling with EQS: Basic concepts, applications, and programming. LEA.

Calvete, E., Gámez-Guadix, M., Orue, I., González-Diez, Z., López de Arroyabe, E., Sampedro, R., Pereira, R., Zubizarreta, A., \& Borrajo, E. (2013). Brief report: The adolescent child-to-parent aggression questionnaire: An examination of aggression against parents in Spanish adolescents. Journal of Adolescence, 36(6), 1077-1081. https://doi.org/10.1016/j.adolescence.2013.08.017

Calvete, E., \& Orue, I. (2016). Violencia filio-parental: Prevalencias y razones para las agresiones contra padres y madres. Behavioral Psychology, 24(3), 481-495.

Calvete, E., Orue, I., \& Gámez-Guadix, M. (2012). Child-to-parent violence: Emotional and behavioral predictors. Journal of Interpersonal Violence, 28(4), 755-772. https://doi.org/10.1177/ 0886260512455869

Calvete, E., Orue, I., \& Gámez-Guadix, M. (2015a). Reciprocal longitudinal associations between substance use and child-to-parent violence in adolescents. Journal of Adolescence, 44, 124-133. https://doi.org/10.1016/j.adolescence.2015.07.015

Calvete, E., Orue, I., Gámez-Guadix, M., \& Bushman, B. J. (2015b). Predictors of child-to-parent aggression: A 3-year longitudinal study. Developmental Psychology, 51, 663. https://doi.org/10. 1037/a0039092

Calvete, E., Orue, I., \& González-Cabrera, J. (2017). Violencia filio parental: Comparando lo que informan los adolescentes y sus progenitores. Revista De Psicología Clínica Con Niños y Adolescentes, 4(1), 9-15.

Calvete, E., Orue, I., \& Sampedro, R. (2011). Violencia filio-parental en la adolescencia: Características ambientales y personales. Infancia y Aprendizaje, 34(3), 349-363. https://doi.org/10.1174/ 021037011797238577

Colins, O. F., Andershed, H., Frogner, L., Lopez-Romero, L., Veen, V., \& Andershed, A. K. (2014). A new measure to assess psychopathic personality in children: The child problematic traits inventory. Journal of Psychopathology and Behavioral Assessment, 36, 4-21. https://doi.org/10.1007/ s10862-013-9385-y

Colins, O. F., Andershed, H., Salekin, R. T., \& Fanti, K. A. (2018). Comparing different approaches for subtyping children with conduct problems: Callous-unemotional traits only versus the multidimensional psychopathy construct. Journal of Psychopathology and Behavioral Assessment, 40(1), 6-15. https://doi. org/10.1007/s10862-018-9653-y

Dawson, J. F. (2018). Interpreting interaction effects. Retrieved June 27, 2020, from http://www.jeremydawson.com/slopes.htm

Del Hoyo-Bilbao, J., Gámez-Guadix, M., \& Calvete, E. (2018a). Corporal punishment by parents and child-to-parent aggression in Spanish adolescents. Anales De Piscología., 34, 108-116. https:// doi.org/10.6018/analesps.34.1.259601

Del Hoyo-Bilbao, J., Gámez-Guadix, M., Orue, I., \& Calvete, E. (2018b). Psychometric properties of the Child-to-Parent Aggression Questionnaire in a clinical sample of adolescents who abuse their parents: Prevalence and gender differences. Violence and Victims., 33, 203-217. https://doi.org/10.1891/0886-6708. VV-D-16-00166

Del Hoyo-Bilbao, J., Orue, I., Gámez-Guadix, M., \& Calvete, E. (2020). Multivariate models of child-to-mother violence and child-to-father violence among adolescents. The European Journal of Psychology Applied to Legal Context, 12, 11-21. https:// doi.org/10.5093/ejpalc2020a2

Fanti, K. A., \& Kimonis, E. R. (2012). Bullying and victimization: The role of conduct problems and psychopathic traits. Journal of Research on Adolescence, 22, 617-631. https://doi.org/10.1111/j. 1532-7795.2012.00809.x 
Fanti, K. A., Frick, P. J., \& Georgiou, S. (2009). Linking callousunemotional traits to instrumental and non-instrumental forms of aggression. Journal of Psychopathology and Behavioral Assessment, 31, 285-298. https://doi.org/10.1007/s10862-008-9111-3

Fanti, K. A., Kyranides, M. N., Lordos, A., Colins, O. F., \& Andershed, H. (2018). Unique and interactive associations of callousunemotional traits, impulsivity and grandiosity with child and adolescent conduct disorder symptoms. Journal of Psychopathology and Behavioral Assessment, 40, 40-49. https://doi.org/ 10.1007/s10862-018-9655-9

Frick, P. J., Bodin, S. D., \& Barry, C. T. (2000). Psychopathic traits and conduct problems in community and clinic-referred samples of children: Further development of the Psychopathy Screening Device. Psychological Assessment, 12(4), 382-393. https://doi.org/10.1037/ 1040-3590.12.4.382

Frick, P. J., Stickle, T. R., Dandreaux, D. M., Farrell, J. M., \& Kimonis, E. R. (2005). Callous-unemotional traits in predicting the severity and stability of conduct problems and delinquency. Journal of Abnormal Child Psychology, 33(4), 471-487. https://doi.org/10. 1007/s10648-005-5728-9

Frick, P. J., \& White, S. F. (2008). Research review: The importance of callous-unemotional traits for developmental models of aggressive and antisocial behavior. Journal of Child Psychology and Psychiatry, 49(4), 359-375. https://doi.org/10.1111/j.1469-7610. 2007.01862.x

Gámez-Guadix, M., \& Calvete, E. (2012). Violencia filio-parental y su asociación con la exposición a la violencia marital y la agresión de padres a hijos. Psicothema, 24, 277-283.

Ghanizadeh, A., \& Jafari, P. (2010). Risk factors of abuse of parents by their ADHD children. European Child \& Adolescent Psychiatry, 19(1), 75-81. https://doi.org/10.1007/s00787-009-0067-y

Hillege, S., Das, J., \& de Ruiter, C. (2010). The youth psychopathic traits inventory: Psychometric properties and its relation to substance use and interpersonal style in a Dutch sample of nonreferred adolescents. Journal of Adolescence, 33(1), 83-91. https://doi.org/10.1016/j.adolescence.2009.05.006

Ibabe, I., \& Bentler, P. (2016). The contribution of family relationships to child-to-parent violence. Journal of Family Violence, 31, 259-269. https://doi.org/10.1007/s10896-015-9764-0

Ibabe, I., \& Jaureguizar, J. (2010). Child-to-parent violence: Profile of abusive adolescents and their families. Journal of Criminal Justice, 38(4), 616-624. https://doi.org/10.1016/j.jcrimjus.2010. 04.034

Jöreskog, K. G., \& Sörbom, D. (2006). LISREL 8.80 for Windows. [Computer Software]. Lincolnwood, IL: Scientific Software International.

Kennedy, T. D., Edmonds, W., Dann, K. T., \& Burnett, K. F. (2010). The clinical and adaptive features of young offenders with histories of child-parent violence. Journal of Family Violence, 25 , 509-520. https://doi.org/10.1007/s10896-010-9312-x.3

Kiire, S. (2017). Psychopathy rather than Machiavellianism or narcissism facilitates intimate partner violence via fast life strategy. Personality and Individual Differences, 104, 401-406. https://doi. org/10.1016/j.paid.2016.08.043

Lilienfeld, S. O. (2018). The multidimensional nature of psychopathy: Five recommendations for research. Journal of Psychopathology and Behavioral Assessment, 40, 79-85. https://doi.org/10.1007/ s10862-018-9657-7

López-Larrañaga, M., \& Orue, I. (2019). Interaction of psychopathic traits in the prediction of cyberbullying behavior. Revista De Psicopatologia y Psicologia Clinica, 24(1), 1-8. https://doi.org/ 10.5944/rppc.23932

Lyons, J., Bell, T., Fréchette, S., \& Romano, E. (2015). Child-toparent violence: Frequency and family correlates. Journal of Family Violence, 30(6), 729-742. https://doi.org/10.1007/ s10896-015-9716-8
Marcus, D. K., John, S. L., \& Edens, J. F. (2004). A taxometric analysis of psychopathic personality. Journal of Abnormal Psychology, 113(4), 626-635. https://doi.org/10.1037/0021-843X.113.4.626

Margolin, G., \& Baucom, B. R. (2014). Adolescents' aggression to parents: Longitudinal links with parents' physical aggression. Journal of Adolescent Health, 55(5), 645-651. https://doi.org/ 10.1016/j.jadohealth.2014.05.008

Marsee, M. A., Silverthorn, P., \& Frick, P. J. (2005). The association of psychopathic traits with aggression and delinquency in non-referred boys and girls. Behavioral Sciences \& the Law, 23, 803-817. https://doi.org/10.1002/bsl.662

Orue, I., \& Andershed, H. (2015). The youth psychopathic traits inventory-short version in Spanish adolescents-factor structure, reliability, and relation with aggression, bullying, and cyber bullying. Journal of Psychopathology and Behavioral Assessment, 37, 563-575. https://doi.org/10.1007/s10862-015-9489-7

Orue, I., \& Calvete, E. (2019). Psychopathic traits and moral disengagement interact to predict bullying and cyberbullying among adolescents. Journal of Interpersonal Violence, 34(11), 23132332. https://doi.org/10.1177/0886260516660302

Orue, I., Calvete, E., \& Gamez-Guadix, M. (2016). Gender moderates the association between psychopathic traits and aggressive behavior in adolescents. Personality and Individual Differences, 94, 266-271. https://doi.org/10.1016/j.paid.2016.01.043

Pagani, L., Tremblay, R., Nagin, D., Zoccolillo, M., Vitaro, F., \& McDuff, P. (2004). Risk factor models for adolescent verbal and physical aggression toward mothers. International Journal of Behavioral Development, 28(6), 528-537. https://doi.org/10. 1080/01650250444000243

Pagani, L., Tremblay, R. E., Nagin, D., Zoccolillo, M., Vitaro, F., \& McDuff, P. (2009). Risk factor models for adolescent verbal and physical aggression toward fathers. Journal of Family Violence, 24(3), 173-182. https://doi.org/10.1007/s10896-008-9216-1

Penney, S. R., \& Moretti, M. M. (2007). The relation of psychopathy to concurrent aggression and antisocial behavior in high-risk adolescent girls and boys. Behavioral Sciences \& the Law, 25(1), 21-41. https://doi.org/10.1002/bsl.715

Pereira, R., \& Bertino, L. (2009). Una comprensión ecológica de la violencia filio-parental. In R. Giraldo \& M. I. Gónzalez (Eds.), Violencia familiar (pp. 226-252). Editorial Universidad del Rosario.

Pereira, R., Loinaz, I., del Hoyo-Bilbao, J., Arrospide, J., Bertino, L., Calvo, A., Montes, Y., \& Gutiérrez, M. M. (2017). Propuesta de definición de violencia filio-parental: Consenso de la sociedad española para el estudio de la violencia filio-parental (SEVIFIP) [Proposal for a definition of filioparental violence: Consensus of the Spanish society for the study of filio-parental violence (SEVIFIP)]. Papeles del Psicólogo, 38, 216-233. https://doi.org/10. 23923/pap.psicol2017.2839

Preacher, K. J., \& Coffman, D. L. (2006). Computing power and minimum sample size for RMSEA [computer software]. Available from http://quantpsy.org/. Accessed 15 June 2021.

Purcell, R., Baksheev, G. N., \& Mullen, P. E. (2014). A descriptive study of juvenile family violence: Data from intervention order applications in a childrens court. International Journal of Law and Psychiatry, 37(6), 558-563. https://doi.org/10.1016/j.ijlp.2014.02.029

Rico, E., Rosado, J., \& Cantón-Cortés, D. (2017). Impulsiveness and child-to-parent violence: The role of aggressor's sex. The Spanish Journal of Psychology, 20(15). https://doi.org/10.1017/sjp. 2017.15

Routt, G., \& Anderson, L. (2011). Adolescent violence towards parents. Journal of Aggression, Maltreatment \& Trauma, 20(1), 1-18. https://doi.org/10.1080/10926771.2011.537595

Salekin, R. T. (2016a). Psychopathy in childhood: Toward better informing the DSM-5 and ICD-11 conduct disorder specifiers. Personality Disorders: Theory, Research, and Treatment, 7(2), 180-191. https://doi.org/10.1037/per0000150 
Salekin, R. T. (2016b). Psychopathy in childhood: why should we care about grandiose-manipulative and daring-impulsive traits? The British Journal of Psychiatry, 209(3), 189-191. https://doi.org/ 10.1192/bjp.bp.115.179051

Salekin, R. T. (2017). Research review: What do we know about psychopathic traits in children? Journal of Child Psychology and Psychiatry, 58(11), 1180-1200. https://doi.org/10.1111/jcpp.12738

Simmons, M., McEwan, T. E., Purcell, R., \& Ogloff, J. R. (2018). Sixty years of child-to-parent abuse research: What we know and where to go. Aggression and Violent Behavior, 28, 31-52. https://doi.org/ 10.1016/j.avb.2017.11.001

The Spanish Society of Epidemiology and the Spanish Society of Family and Community Medicine. (2000). Una propuesta de medida de la clase social. Atención Primaria, 25(5), 350-363. https://doi. org/10.1016/S0212-6567(00)78518-0

Stickle, T. R., Marini, V. A., \& Thomas, J. N. (2012). Gender Differences in Psychopathic Traits, Types, and Correlates of Aggression Among Adjudicated Youth. Journal of Abnormal Child Psychology, 40, 513-525. https://doi.org/10.1007/s10802-011-9588-1
Strom, K. J., Warner, T. D., Tichavsky, L., \& Zahn, M. A. (2014). Policing juveniles: Domestic violence arrest policies, gender, and police response to child-parent violence. Crime \& Delinquency, 60(3), 427-450. https://doi.org/10.1177/0011128710376293

Somma, A., Andershed, H., Borroni, S., Salekin, R. T., \& Fossati, A. (2018). Psychopathic personality traits in relation to self-report delinquency in adolescence: Should we mind about interaction effects? Journal of Psychopathology and Behavioral Assessment, 40(1), 69-78. https://doi.org/10.1007/s10862-018-9658-6

Van Baardewijk, Y., Andershed, H., Stegge, H., Nilsson, K. W., Scholte, E., \& Vermeiren, R. (2010). Development and tests of short versions of the youth psychopathic traits inventory and the youth psychopathic traits inventory-child version. European Journal of Psychological Assessment, 26(2), 122-128. https://doi.org/ 10.1027/1015-5759/a00001

Publisher's Note Springer Nature remains neutral with regard to jurisdictional claims in published maps and institutional affiliations. 